\title{
Efecto de dos dosis de cipionato de estradiol y del rango horario para la IATF sobre la tasa de preñez en vaquillonas"
}

\author{
Madero, S. ; De Dominicis, O. ; Cantallops, F.'; Uslenghi, G. ${ }^{2,3}$; Callejas, S. ${ }^{3}$ \\ ${ }^{1}$ Actividad privada, Provincia de Buenos Aires (Argentina), ${ }^{2}$ Becario Posgrado CONICET, ${ }^{3}$ Área de \\ Reproducción, Facultad Ciencias Veterinarias UNCPBA, Tandil (Argentina). \\ E-mail: callejas@vet.unicen.edu.ar
}

\begin{abstract}
Resumen
Madero, S.; De Dominicis, O.; Cantallops, F.; Uslenghi, G.; Callejas, S.: Efecto de dos dosis de cipionato de estradiol y del rango horario para la IATF sobre la tasa de preñez en vaquillonas. Rev. vet. 23: 1, 46-48, 2012. El objetivo de este trabajo fue evaluar el efecto de dos dosis de cipionato de estradiol (CPE) y dos intervalos para realizar la inseminación, sobre el porcentaje de preñez a la inseminación artificial a tiempo fijo (IATF). Se utilizaron 326 vaquillonas de 24 meses, Angus negro (AAN) y colorado (AAC), de condición corporal (media \pm de) $3,7 \pm 0,3$ (escala 1-5). El día 0 se les colocó un dispositivo intravaginal con progesterona (DISP, DIB 0,5, Syntex) más $2 \mathrm{mg}$ de benzoato de estradiol (Estradiol 10, Río de Janeiro) im. El día 8 se retiró el DISP, se administró im $150 \mu \mathrm{g}$ de $\mathrm{D}(+)$ Cloprostenol (Arsaprost, ARSA) y los animales recibieron aleatoriamente 0,5 o $1 \mathrm{mg}$ de cipionato de estradiol (Grupo CPE05 y CPE1, respectivamente). El día 10 las vaquillonas fueron inseminadas, dentro de cada tratamiento, a las 48-52 h o 53-58 h post-retiro de los dispositivos. Se utilizó semen de dos toros (A y B), los cuales estuvieron distribuidos aleatoriamente dentro de cada raza, tratamiento y rango horario. El diagnóstico de gestación se realizó por palpación transrrectal a los 63 días de realizada la IATF. Se evaluó el efecto raza (AAN y AAC), tratamiento (CPE05 o CPE1), toro (A y B), hora de IATF (48-52 y 53-58) y las interacciones dobles sobre el porcentaje de preñez. Se utilizó el PROC CATMOD del SAS, fijando un nivel de confianza del $95 \%(\alpha=0,05)$. No se observaron efectos significativos de la raza (AAC: $49,1 \%$; AAN: $57,1 \%$ ), tratamiento (CPE05: 51,6\%; CPE1: 51,5\%), toro (A: 54,6\%, B: 48,5\%), hora de IATF (48-52: $54,4 \% ; 53-58: 48,8 \%$ ) o sus interacciones dobles ( $p>0,05)$.
\end{abstract}

Palabras clave: vaquillona, IATF, estradiol cipionato, progesterona, rango horario, porcentaje de preñez.

\begin{abstract}
Madero, S.; De Dominicis, O.; Cantallops, F.; Uslenghi, G.; Callejas, S.: Effect of two doses of estradiol cypionate and hourly rank for FTAI on pregnancy rate in heifers. Rev. vet. 23: 1, 46-48, 2012. The objective of this work was to evaluate the effect of two doses of estradiol cypionate (ECP) and two intervals for insemination upon pregnancy rate at FTAI. A total of 326 black and red Angus heifers (BA and RA) with 24 months-old and a body score condition of $3.7 \pm 0.3(\mathrm{~m} \pm \mathrm{ed})$ participated on the trial. On day 0 heifers received a progesterone intravaginal device (DIB, $0.5 \mathrm{~g}$, Syntex) plus $2 \mathrm{mg}$ estradiol benzoate (Estradiol 10, Río de Janeiro). On day 8, the intravaginal device was removed, $150 \mu \mathrm{g}$ de $\mathrm{D}(+)$ Cloprostenol (Arsaprost, ARSA) were administered and animals were randomized to receive $0.5 \mathrm{or} 1 \mathrm{mg}$ ECP (group ECP05 and ECP1 respectively). On day 10 heifers received FTAI, for each treatment, at $48-52 \mathrm{~h}$ or $53-58 \mathrm{~h}$ after device removal. Semen of two bull (A and B) was used, which was distributed aleatory in each breed, treatment, and time range. Pregnancy diagnosis was performed by rectum palpation on day 63 after FTAI. The effect of breed (BA and RA), treatments (ECP05 and ECP1), bull (A and B), time range (48-52 y 53-58 h) and their interactions upon pregnancy rate at FTAI were evaluated. Variables were analyzed using PROC CATMOD of SAS, significance was indicated by a probability of $\mathrm{p}<0.05$. There were no effects of breed (RA: 49.1\%; BA: 57.1\%), treatment (ECP05: 51.6\%; ECP1: 51.5\%), bull (A: 54.6\%, B: 48.5\%), time range (48-52: 54.4\%; 53-58: 48.8\%) and their interactions ( $\mathrm{p}>0.05)$ upon pregnancy rates at FTAI.
\end{abstract}

Key words: heifer, FTAI, estradiol cypionate, progesterone, time range, pregnancy rate. 


\section{INTRODUCCIÓN}

El tratamiento hormonal que se utiliza tradicionalmente para implementar una inseminación artificial a tiempo fijo (IATF) ${ }^{3}$ en rodeos comerciales requiere del encierre de los animales en cuatro oportunidades, y uno de ellas consiste en inyectar benzoato de estradiol (BE) $24 \mathrm{~h}$ posteriores al retiro de los dispositivos intravaginales ${ }^{4,5}$. Desde hace varios años se trabaja para reducir el número de encierres y facilitar el manejo de los animales; en este sentido el cipionato de estradiol (CPE) aplicado al retirar los dispositivos es el tratamiento más utilizado, ya que sincroniza las ovulaciones de manera similar a la aplicación de $\mathrm{BE}^{2}$.

El momento en que se realiza la inseminación es muy importante. En tal sentido, se afirma que la IA se debería realizar 8-12 h previas a la ovulación ${ }^{12}$, pese a que otros autores consideran ideal 12 a $24 \mathrm{~h}^{2}$, siendo adecuada 16 $\mathrm{h}$ antes de la ovulación para lograr óptimos porcentajes de preñez. De esta manera, considerando que la ovulación se produce en promedio a las $70 \mathrm{~h}$ post retiro de los dispositivos ${ }^{5}$, se determina que la IATF podría realizarse 8 a $20 \mathrm{~h}$ antes de la ovulación, lo que equivale a un rango horario de $50 \mathrm{a} 62 \mathrm{~h}$ post retiro de los dispositivos. Comúnmente se obtienen buenos resultados de preñez inseminando a las $54-58 \mathrm{~h}$ post retiro, utilizando el tratamiento tradicional ${ }^{10}$. Sin embargo, hay cierta evidencia que en animales tratados con CPE al retiro, algunas ovulaciones se producen antes del momento de la IATF, lo que podría afectar negativamente la preñez ${ }^{7}$.

Por otro lado, la dosis de CPE con que se ha trabajado en vaquillonas ha sido de $0,5 \mathrm{mg}^{7} \mathrm{y}$ en vacas de $1 \mathrm{mg}{ }^{14}$; por lo que surge de interés evaluar estas dosis en vaquillonas y verificar si existe relación con el rango horario en que se realiza la IATF.

Por lo tanto, el objetivo del presente trabajo fue evaluar el efecto de administrar dos dosis de CPE al retirar un dispositivo intravaginal con progesterona en dos intervalos para realizar la IATF, sobre el porcentaje de preñez post tratamiento.

\section{MATERIAL Y MÉTODOS}

Lugar, animales, alimentación. El trabajo se realizó en un establecimiento de producción agropecuaria ubicado en el partido de General Pinto, Provincia de Buenos Aires, Argentina. Se utilizaron 326 vaquillonas de 24 meses de edad, Aberdeen Angus negro (AAN) y colorado (AAC), con una condición corporal

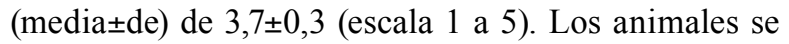
alimentaron de pasturas con raigrás y trébol blanco durante el período experimental, con libre acceso al agua de bebida. Además, 2,5 meses previos a la IATF y hasta dicho momento recibieron una suplementación de 3 $\mathrm{kg}$ de maíz entero y $1 \mathrm{~kg}$ de sojilla por día/animal.

Tratamientos y servicio. El día 0 se le colocó a todas las vaquillonas un dispositivo (DISP) intravaginal con progesterona (DIB 0,5 g Syntex, Argentina) más una inyección intramuscular (im) de $2 \mathrm{mg}$ de BE (Es-
Tabla 1. Porcentajes de preñez obtenidos.

\begin{tabular}{cccc}
\hline hora IATF & tratamiento & \multicolumn{2}{c}{ preñez (\%) } \\
\hline \multirow{2}{*}{$48-52$} & CPE05 & $57,3(47 / 82)$ & 54,4 \\
& CPE1 & $51,3(39 / 76)$ & $(86 / 158)$ \\
\hline \multirow{2}{*}{$53-58$} & CPE05 & $45,6(36 / 79)$ & 48,8 \\
& CPE1 & $51,7(46 / 89)$ & $(82 / 168)$ \\
\hline \multirow{2}{*}{$48-58$} & CPE05 & $51,6(83 / 161)$ & 51,5 \\
& CPE1 & $51,5(85 / 165)$ & $(168 / 326)$ \\
\hline
\end{tabular}

Datos de vaquillonas Angus que recibieron un dispositivo intravaginal con progesterona combinado con dos dosis de Cipionato de Estradiol para sincronizar la ovulación y fueron inseminadas (IATF) en dos rangos horarios post dispositivo.

tradiol 10, Río de Janeiro, Argentina). El día 8 se retiró el DISP, se administró im $150 \mu \mathrm{g}$ de $\mathrm{D}(+)$ Cloprostenol (Arsaprost, ARSA, Argentina) y los animales recibieron aleatoriamente 0,5 o $1 \mathrm{mg}$ de CPE (grupos CPE05 y CPE1, respectivamente). El día 10 se realizó la IATF en todas las vaquillonas, a las $48-52 \mathrm{~h}$ o a las $53-58 \mathrm{~h}$ de retirados los dispositivos, aleatoriamente distribuidas dentro de cada grupo, quedando conformados cuatro grupos (CPE05:48-52 h; CPE05:53-58 h; CPE1:48-52 h; CPE1:53-58 h). Para la IATF se utilizó semen congelado/descongelado en pajuelas de $0,5 \mathrm{ml}$ proveniente de 2 toros (A y B) de probada fertilidad, cuyos análisis de laboratorio fueron para el toro A: motilidad progresiva (MP) a las horas 0 y $2,40 \%$ y $40 \%$, vigor: 3 en ambas lecturas, morfología: $88 \%$ normales, número de espermatozoides con MP (NEMP): 6.520 .000 por pajuela; toro B: MP: $50 \%$ y $45 \%$ a las horas 0 y 2; vigor: 3 en ambas lecturas, morfología: 87\% normales, NEMP: 14.800.000 por pajuela). Los toros estuvieron distribuidos aleatoriamente dentro de cada raza, tratamiento y rango horario de IATF.

Diagnóstico de gestación. Se realizó mediante palpación transrectal a los 63 días posteriores a la IATF.

Análisis estadístico. Se evaluó el efecto de la raza (AAN y AAC), el tratamiento (CPE05 o CPE1), el toro (A y B), el rango horario para la IATF (48-52 y 53-58) y las interacciones dobles sobre el porcentaje de preñez a la IATF. Se utilizó el PROC CATMOD del SAS, fijando un nivel de confianza del $95 \%(\alpha=0,05)$.

\section{RESULTADOS}

No se observaron efectos significativos de la raza (AAC: 49,1\%; AAN: 57,1\%), del tratamiento (Tabla 1), del toro (A: $54,6 \%$, B: 48,5\%), del rango horario para la IATF (Tabla 1) o de sus interacciones dobles ( $p>0,05)$.

\section{DISCUSIÓN}

La utilización de CPE al retiro de los dispositivos ha permitido disminuir el número de encierres de los animales, obteniendo porcentajes de preñez equivalentes al BE aplicado $24 \mathrm{~h}$ posteriores ${ }^{11,13}$. Así, bajas dosis de CPE han sido utilizadas para sincronizar las ovulaciones en ganado lechero ${ }^{1}$, como así también se ha 
utilizado con éxito en protocolos de IATF para vaquillonas de carne ${ }^{8}$. En el presente trabajo, el porcentaje de preñez a la IATF no fue afectado significativamente por el tratamiento de sincronización de la ovulación. La administración de $0,5 \mathrm{mg}$ de CPE demostró ser igualmente efectiva que la dosis de $1 \mathrm{mg}$ para sincronizar las ovulaciones y obtener adecuados porcentajes de preñez.

Otros investigadores evaluaron las tasas de preñez con diferentes dosis de $\mathrm{CPE}^{6}$. Utilizaron 667 vaquillonas de 15 meses de edad, las cuales recibieron dos tratamientos: 0,5 o $1 \mathrm{mg}$ de CPE al momento de retirar los dispositivos. La IATF se realizó a las $52-56 \mathrm{~h}$ posteriores al retiro de los dispositivos y las tasas de preñez obtenidas fueron $55,7 \%$ y $57,4 \%$ para los grupos $0,5 \mathrm{mg}$ y $1 \mathrm{mg}$ de CPE respectivamente. En este trabajo no se encontraron diferencias significativas entre tratamientos de inducción de la ovulación, demostrando que ambas dosis son igualmente efectivas.

Con respecto al momento de la inseminación, se ha demostrado que la IATF puede realizarse en un rango horario de 48 a 54 h post retiro de los dispositivos sin afectar los porcentajes de preñez cuando se utiliza el tratamiento tradicional ${ }^{2}$. En el presente trabajo fueron utilizadas dos dosis de CPE y dos rangos horarios para realizar la IATF, los mismos fueron definidos entre las 48 y $52 \mathrm{~h}$ y entre las 53 y $58 \mathrm{~h}$. No fueron observadas diferencias significativas entre los rangos para realizar la IATF sobre el porcentaje de preñez.

En otro ensayo se realizaron dos experimentos para evaluar el efecto del momento de la IATF sobre las tasas de preñez en vaquillonas tratadas con CPE como inductor de la ovulación ${ }^{9}$. Se comprobó que cuando se utilizan dispositivos de segundo uso, equivalentes a los utilizados en el presente trabajo $(0,5 \mathrm{~g}$ de progesterona), no existen diferencias en el porcentaje de preñez realizando la IATF entre las 47 y $59 \mathrm{~h}$ post retiro de los dispositivos. Estos hechos coinciden con los resultados aquí obtenidos. En contraposición, dichos investigadores obtuvieron menor porcentaje de preñez en animales tratados con dispositivos nuevos (1 $\mathrm{g}$ de progesterona) cuando la IATF se realizó a las 47-48 h, no así cuando se realizó entre las 53 y 59 h. Futuros trabajos deberán profundizar los estudios en este aspecto, para explicar las divergencias observadas.

Se concluye que la administración de $0,5 \mathrm{mg}$ o 1 mg de CPE en el momento de retirar un dispositivo intravaginal con progesterona no afecta el porcentaje de preñez, pudiendo realizar la IATF entre las 48 y $58 \mathrm{~h}$ posteriores a su administración.

\section{REFERENCIAS}

1. Ambrose JD, Kastelic JP, Rajamahendran R, Aali M, Dinn N. 2005. Progesterone (CIDR)-based timed AI protocols using GnRH, porcine LH or estradiol cypionate for dairy heifers: Ovarian and endocrine responses and pregnancy rates. Theriogenology 64: 1457-1474.

2. Ayres H, Martins CM, Ferreira RM, Mello JE, Dominguez JH, Souza AH, Valentin R, Santos IC, Baruselli
PS. 2008. Effect of timing of estradiol benzoate administration upon synchronization of ovulation in suckling Nelore cows (Bos indicus) treated with a progesteronereleasing intravaginal device. Anim Reprod Sci 109: 77-87.

3. Bó GA, Adams GP, Pierson RA, Mapletoft RJ. 1995. Exogenous control of follicular wave emergence in cattle. Theriogenology 43: 31-40.

4. Bó GA, Baruselli PS, Moreno D, Cutaia L, Caccia M, Tríbulo R. 2002. The control of follicular wave development for self appointed embryo transfer programs in cattle. Theriogenology 57: 53-72.

5. Bó GA, Baruselli PS, Martínez MF. 2003. Pattern and manipulation of follicular development in Bos indicus cattle. Anim Reprod Sci 78: 307-326.

6. Chesta P, Brandan A, Cuestas G, Quiñones G, Lozano P, Tríbulo H. 2009. Evaluación de diferentes dosis de cipionato de estradiol sobre la tasa de preñez en inseminación artificial a tiempo fijo en vaquillonas de 15 meses de edad. Anales VIII Simp Intern Reprod Anim IRAC, Córdoba (Argentina), CD N 13.

7. Colazo MG, Kastelic JP, Mapletoft RJ. 2003. Effects of estradiol cypionate (ECP) on ovarian follicular dynamics, synchrony of ovulation, and fertility in CIDR-based, fixed-time AI programs in beef heifers. Theriogenology 60: 855-865.

8. Colazo MG, Kastelic JP, Martinez MF, Whittaker PR, Wilde R, Ambrose JD, Corbett R, Mapletoft RJ. 2004. Fertility following fixed time AI in CIDR-treated beef heifers given GnRH or estradiol cypionate and fed diets supplemented with flax seed or sunflower seed. Theriogenology 61: 1115-1124.

9. Cutaia L, Ramos M, Chesta P, Bó G. 2009. Efecto del momento de la IATF en vaquillonas de carne cruza cebú tratadas con dispositivos con progesterona y ECP como inductor de la ovulación. Anales VIII Simp Intern Reprod Anim IRAC, Córdoba (Argentina), CD N 18.

10. Fernandes P, Teixeira AB, Crocci JA, Barros CM. 2001. Timed artificial insemination in beef cattle using GnRH agonist, PGF2alpha and estradiol benzoate (EB). Theriogenology 55: 1521-1532.

11. MacMillan KL, Segwagwe BE, Pino CS. 2003. Associations between the manipulation of patterns of follicular development and fertility in cattle. Anim Reprod Sci 78: 327-344.

12. Mapletoft RJ, Bó GA, Martínez M, Colazo M, Caccia M, Adams GP. 1999. Control del desarrollo folicular y su uso en programas de inseminación artificial a tiempo fijo en ganado de carne. Anales III Simp Intern Reprod Anim IRAC, Córdoba (Argentina), p. 51-69.

13. Meneghetti M, Sá-Filho OG, Peres RF, Lamb GC, Vasconcelos JL. 2009. Fixed time artificial insemination with estradiol and progesterone for Bos indicus cows I: Basis for development of protocols. Theriogenology 72: 179-189.

14. Sá Filho MF, Santos JE, Ferreira RM, Sales JN, Baruselli PS. 2011. Importance of estrus on pregnancy per insemination in suckled Bos indicus cows submitted to estradiol/progesterone-based timed insemination protocols. Theriogenology 76: 455-463. 